\title{
Laparoscopic radical hysterectomy and tension-free vaginal tape-obturator (TVT-0) combined to treat cervical cancer complicating pelvic organ prolapse (POP) and stress urinary incontinence (SUI): A case report
}

\section{Yu Ran}

Chongqing Medical University

\section{Qiang Yi}

Chongqing Medical University

Cong Li ( $\nabla$ tg202010@163.com )

Chongqing Medical University https://orcid.org/0000-0002-8234-2121

Qin Zhou

Chongqing Medical University

\section{Case report}

Keywords: Pelvic organ prolapse (POP), stress urinary incontinence (SUI), cervical cancer, surgery-based treatment, tension-free vaginal tape-obturator (TVT-0), quality of life (QOL), human papillomavirus (HPV) infection

Posted Date: April 16th, 2021

DOl: https://doi.org/10.21203/rs.3.rs-265039/v2

License: (c) (i) This work is licensed under a Creative Commons Attribution 4.0 International License. Read Full License 


\section{Abstract}

Background: Combined cervical cancer and pelvic organ prolapse (POP) is extremely uncommon and there is no standard treatment guideline for the complex comorbidity. We attempted to provide a treatment strategy and explore possible reasons for the comorbidities.

Case presentation: We presented a 62-year-old patient seeking treatment for postmenopausal tissue prolapse and leakage of urine. Physical examination revealed procidentia with a $2.5 \mathrm{~cm}$ papillary and erosive lesion that was pathologically confirmed as cervical squamous cell carcinoma in stage lb2. Clinical diagnosis included cervical squamous cell carcinoma in stage lb2, prolapse of the anterior and posterior walls of the vagina and uterine, stress urinary incontinence (SUI). Then she was performed radical surgery for cervical cancer and tension-free vaginal tape-obturator (TVT-O). The urine function was good after operation. The surgery solved two diseases, which improved the quality of life (QOL) of patient and radically cured cervical malignancies at the same time.

Conclusions: Surgical-based treatment seems to be more suitable that can cure the two diseases and improve the QOL. We should pay attention to the potential high incidence of HPV infection and HPVrelated cervical cancer so that we should not ignore the screening for cervical cancer in the prolapse group and initiate surgery treatment strategies of cervical cancer or pre-cancerous lesions as early as possible to reach an optimal outcome of patient.

\section{Background}

The incidence of cervical cancer in the elderly, especially in developing countries such as China, is still high [1]. In recent years the early diagnosis rate has further increased due to the popularization of cervical screening [2]. Pelvic organ prolapses (POP) including the uterine or vaginal procidentia and stress urinary incontinence (SUI), is of high-incidence in the elderly population, estimated to occur in $40-60 \%$ of women aged 60-69 years [3], which has a significant effect on quality of life (QOL). However, cervical cancer combined with POP is rare, with an incidence of only $0.14-1 \%[4]$ so that there is no standard therapeutic method. One of the treatment choices is using pessary for organ prolapse after radiotherapy for cervical cancer. Surgery-based treatment is also an option that conducts radical surgical treatment while performing pelvic floor reconstruction with biological mesh, which is clinically feasible for the treatment of malignant tumors and improvement of QOL at the same time, especially for early cervical cancer. The potential high incidence of human papillomavirus (HPV) infection may be the most important factor of the cervical cancer [5] in genital prolapse population.

\section{Case Presentation}

A 62-year-old patient with a 30-year history of POP used pessary for 10 years, after anterior and posterior colporrhaphy which relieved her symptom. But she complained of a worsening pelvic pressure that the pessary could not prevent the prolapsing uterus and SUI as well, with occasional small vaginal bleeding 
for 3 months. She was in good general condition and had been menopausal for 12 years. She had four pregnancies and two labors (all under vaginal delivery 34 and 38 years ago respectively), and induced labor twice in 7 months of pregnancy owning to intrauterine death and in 4 months of pregnancy for personal factors. Family history and personal history have nothing special. Physical examination revealed anterior vaginal wall prolapse and bladder partialbulge. The cervix and posterior wall of the vagina were located at the orificium vagina (POP-Q [Pelvic Organ Prolapse Quantification] system score was showed in Fig. 1.), with a $2.5 \mathrm{~cm}$ severe cervical erosion that was papillary and containing bleeding in the posterior lip of cervix. Thinprep cytologic test (TCT) indicated Atypical Squamous Cells of Undetermined Significance (AS-CUS), HPV 16 DNA and E6E7(+). Biopsy of the orientation of cervical 7 o'clock and endocervical curettage under colposcopy confirmed a carcinoma in situ squamous cell carcinoma of the cervix, focal invasive carcinoma (could not definite the depth because of the superficial biopsy), high grade squamous intraepithelial lesion (HSIL) in cervical canal.

She was performed laparoscopic radical hysterectomy, bilateral salpingo-oophorectomy and pelvic lymphadenectomy. After radical hysterectomy we rechecked the prolapse of vaginal wall. No obvious prolapse was found in anterior and posterior wall, only tension-free vaginal tape-obturator (TVT-O) was operated. The pathological result showed no obvious abnormality besides the cervix as described above. The postoperative pathological analysis demonstrated HSIL affected gland in cervix and HSIL in vaginal wall with no infiltrating or involving foci of carcinoma, negative surgical margin, and absence of metastatic carcinoma in pelvic lymph nodes $(0 / 40)$. Therefore, the final diagnosis was cervical squamous carcinoma staged Ib2. The patient recovered well after the operation. The pelvic floor anatomical structure was restored without procidentia and leakage of urine. No additional treatment was given.

\section{Discussion And Conclusions}

It is well known that the occurrence of cervical cancer is associated with persistent HPV infection [5]. However, we do not know if the uterine and vaginal prolapse increase the risk of HPV infection. In other words, what's the incidence of procidentia complained with HPV infection and the cervical cancer in postmenopausal women? In this case, the cervical cancer in our index patient was discovered by accident through routine preoperative cervical cancer screening that found HPV 16 infection and was confirmed by cervical biopsy. Maybe prolapse would increase the rate of HPV infection and cancer rate, but no more data supported. On the one hand, the majority of the people cannot take steps for contraception(especially use condoms) if they have sexual life., the changes of vaginal local microenvironment and weakened immunity, that is deteriorated in postmenopausal women because of the deficiency of estrogen, in addition with some systemic complications such as diabetes mellitus lead to increasing non-specific infection [7]. In our case the patient had the history of using pessary for around 10 years before she came to our hospital. Despite that the pessary, which is placed into the vagina to restore pelvic anatomy and decrease prolapse symptoms, is the only available, non-surgical first-line intervention especially for high-risk surgical candidates with POP that can relieve prolapse and urinary symptoms, it can cause serious complications such as bleeding and ulceration [8] that our index patient 
also appeared. Although the pessary itself does not cause cervical cancer, the underlying increasing chance of nonspecific infection above-mentioned with the repeated take-out and put-in of the pessary may be a considerable factor for the progression of disease, so as the cervical carcinogenesis and precancerous changes of the vagina that confirmed through postoperative pathological analysis.

The treatment strategies for invasive cervical carcinoma are often dictated by the clinical stage, based on presence of distant metastasis and patient's performance status. For cervical squamous cancer staging $\mathrm{Ib}_{2}$, in addition to radical surgical treatment, pelvic external beam radiation therapy (EBRT) plus with brachytherapy is also an option especially for patients who have contraindications to surgery or refuse surgery according to 2020 NCCN Guidelines of Cervical Cancer [9]. Moreover, due to the degradation of immune and physiological functions in elderly patients, reducing tolerance to wide surgery range and anesthesia, the long postoperative recovery time, prolonged start time of adjuvant treatment, the appearance of postoperative complications obstacle to the completion of adjuvant treatment, all these factors ultimately affected the outcome of treatment. Therefore, surgical options for elderly patients are usually conservative. There are many treatment modalities for POP, include pessary, reconstructive pelvic surgery with mesh, sacral colpopexy, colocalizes [10] and autologous tissue repair [11]. Treatment options that were reported of the complex comorbidities in recent years include pessary followed by perineorrhaphy and chemoradiotherapy [12], vaginal hysterectomy with pelvic node dissection followed by radiation therapy [13], total vaginal hysterectomy, trans ventral cancer staging and intraabdominal uterosacral ligament suspension [14], radical hysterectomy and immediate sacral colpopexy with an autologous fascia Lata (RISA) graft [15], even palliative chemotherapy (combined terminal cervical carcinoma) [16]. However, the surgical-based treatment in our index patient seemed to be a more favorable treatment strategy. Firstly, the radical surgery cervical solved the cervical cancer and prolapse of middle pelvic cavity and the following TVT-O relieved the urinary symptoms. By virtue of our large enough resection extent that included $4-5 \mathrm{~cm}$ vaginal wall and subsequent suture of vaginal residue, the remaining vagina of the patient was very short, which had addressed the prolapse of vaginal wall to some extent. Secondly, tumor regression and organ motion can affect the radiation dose, and cystorectocele may increase the risk of visceral radiation injury that lowers QOL if we choose radiotherapy or concurrent chemoradiotherapy, although it may be less invasive [15]. Finally, surgery-based treatment may have a positive effect on survival outcome according to the past two systematic reviews $[4,17]$. Therefore, it is pivotal to gain the availability of surgical opportunities. Besides the patient's general condition that tolerate the risks of surgery and anesthesia, the early detection of cervical cancer or precancerous lesions is more important.

We can take some measures to prevent the occurrence of cervical cancer in POP population that seems more important than treatment. On the one hand, we should popularize vaccination against cancer in young age and the use of condoms in women with postmenopausal prolapse during the sexual activity, appropriate topical application of estrogen to improve vaginal immunity that reduce persistent HPV infection. On the other hand, cervical cancer screening in the population of procidentia could promote early detection and treatment of precancerous lesions. 
In summary, combined cervical cancer and POP is an extremely rare clinical entity and there are no clear treatment recommendations. Surgical-based treatment seems to be more suitable which can cure the two diseases and improve the QOL. We should pay attention to the potential high incidence of HPV infection and HPV-related cervical cancer by screening for cervical cancer in the prolapse group, and initiate standard treatment strategies of cervical cancer or pre-cancerous lesions as early as possible to reach an optimal outcome of patient.

\section{Abbreviations}

POP: pelvic organ prolapses; SUI: stress urinary incontinence; TVT-O: tension-free vaginal tape-obturator; QOL: quality of life; POP-Q: Pelvic Organ Prolapse Quantification; HPV: human papillomavirus; TCT:

Thinprep cytologic test; AS-CUS: Atypical Squamous Cells of Undetermined Significance; HSIL: high grade squamous intraepithelial lesion; EBRT: external beam radiation therapy.

\section{Declarations}

Ethics approval and consent to participateNot applicable.

\section{Consent for publication}

Written informed consent was obtained from the patient for publication in the appropriate journal.

\section{Acknowledgements}

Not applicable.

\section{Availability of data and materials}

Not applicable.

\section{Competing interests}

The authors declare that there is no conflict of interest.

\section{Funding}

No funding supported.

\section{Author contributions}

Qiang Yi collected patient information and searched literature related, Yu Ran and Cong Li were responsible for manuscript writing, Qin Zhou critically reviewed the manuscript and pproved the final manuscript as submitted. 


\section{References}

1. Chen WQ, Zheng RS, Baade PD, et al. Cancer Statistics in China, 2015. Ca Cancer J Clin. 2016;66(2):115-32.

2. Venkatas J, Singh M. Cervical cancer. a meta-analysis, therapy and future of nanomedicine. Ecancermedicalscience. 2020;24;14:1111.

3. Wu JM, Vaughan CP, Goode PS, et al. Prevalence and trends of symptomatic pelvic floor disorders in U.S. women. Obstet Gynecol. 2014;123:141-8.

4. Matsuo K, Fullerton ME, Moeini A. Treatment patterns and survival outcomes in patients with cervical cancer complicated by complete uterine prolapse: a systematic review of literature. Int Urogynecol J. 2016;27:29-38.

5. Bowden SJ, Bodinier B, Kalliala I, et al. Genetic variation in cervical preinvasive and invasive disease: a genome-wide association study. Lancet Oncol. 2021 Apr;22(4):548-57.

6. Bump RC, Mattiasson A, Bø K, Brubaker LP, et al. The standardization of terminology of female pelvic organ prolapse and pelvic floor dysfunction. Am J Obstet Gynecol. 1996 Jul;175(1):10-7.

7. Clabaut M, Suet A, Racine PJ, et al. Effect of $17 \beta$-estradiol on a human vaginal Lactobacillus crispatus strain. Sci Rep. 2021;11(1):7133.

8. Bugge C, Adams EJ, Gopinath D, et al. Pessaries (mechanical devices) for managing pelvic organ prolapse in women. Cochrane Database Syst Rev. 2020;18:11:CD004010.

9. Abu-Rustum NR, Yashar CM, Bean S, et al. NCCN Guidelines Insights: Cervical Cancer, Version 1.2020. J Natl Compr Canc Netw. 2020;18(6):660-6.

10. American College of Obstetricians and Gynecologists' Committee on Practice Bulletins-Gynecology and American Urogynecologic Society. Pelvic Organ Prolapse: ACOG Practice Bulletin, Number 214. Obstet Gynecol. 2019;134(5):e126-42.

11. NICE Guidance. - Urinary incontinence and pelvic organ prolapse in women: management: ( $)$ NICE (2019) Urinary incontinence and pelvic organ prolapse in women: management. BJU Int. 2019;123(5):777-803.

12. Dawkins JC, Lewis GK, Toy EP. Cervical cancer complicating pelvic organ prolapse, and use of a pessary to restore anatomy for optimal radiation: A case report. Gynecol Oncol Rep. 2018;16:26:146.

13. Pardal C, Correia C, Serrano P. Carcinoma of the cervix complicating a genital.

14. prolapse. BMJ Case Rep. 2015; 2015: bcr2015209580.

15. Chung CP, Lee SJ, Wakabayashi MT. Uterine and cervical cancer with irreducible pelvic organ prolapse. Am J Obstet Gynecol. 2018;219(6):621-2.

16. Ota Y, Suzuki Y, Matsunaga T, et al. New combined surgery for cervical cancer complicated by pelvic organ prolapse using autologous fascia lata: A case report. Clin Case Rep. 2020; 20;8(8):1382-1386. 
17. El-Abbassi S, El-Majjaoui S, El-Kacemi H, et al. Complete genital prolapse with cervix carcinoma: a case report and literature review. Arch Can Res. 2017;5:2.

18. Kahn RM, Gordhandas S, Craig K, et al. Cervical carcinoma in the setting of uterovaginal prolapse: comparing standard versus tailored management. Ecancermedicalscience. 2020;14:1043.

\section{Figures}

\begin{tabular}{|l|l|l|}
\hline $\mathrm{Aa}(+1)$ & $\mathrm{Ba}(-1)$ & $\mathrm{C}(0)$ \\
\hline $\mathrm{gh}(6)$ & $\mathrm{pb}(2)$ & $\mathrm{TvL}(8)$ \\
\hline $\mathrm{Ap}(-1)$ & $\mathrm{Bp}(-3)$ & $\mathrm{D}(-2)$ \\
\hline
\end{tabular}

\section{Figure 1}

The POP-Q score of the index patient. From POP-Q score, anterior pelvic prolapse degree II (including anterior vaginal wall prolapse and bladder), middle and posterior pelvic protrusion I degree. Since patient's incontrollable urination, stress urinary incontinence was also diagnosed. POP-Q :Pelvic Organ Prolapse Quantification; Aa, $3 \mathrm{~cm}$ proximal to the external urethral meatus; $\mathrm{Ba}$, most prolapsed portion of the anterior vaginal wall; $\mathrm{C}$, leading edge of the cervix or vaginal cuff; gh, middle of the urethral meatus to the midline of the posterior hymen; $\mathrm{pb}$, middle of the posterior hymen to the middle of the anal opening; $\mathrm{TvL}$, maximum depth of the vagina with prolapse reduced; $\mathrm{Ap}, 3 \mathrm{~cm}$ proximal to the posterior hymen; $\mathrm{Bp}$, most prolapsed portion of the posterior vaginal wall; D, posterior fornix in a woman who has a cervix[6]. 


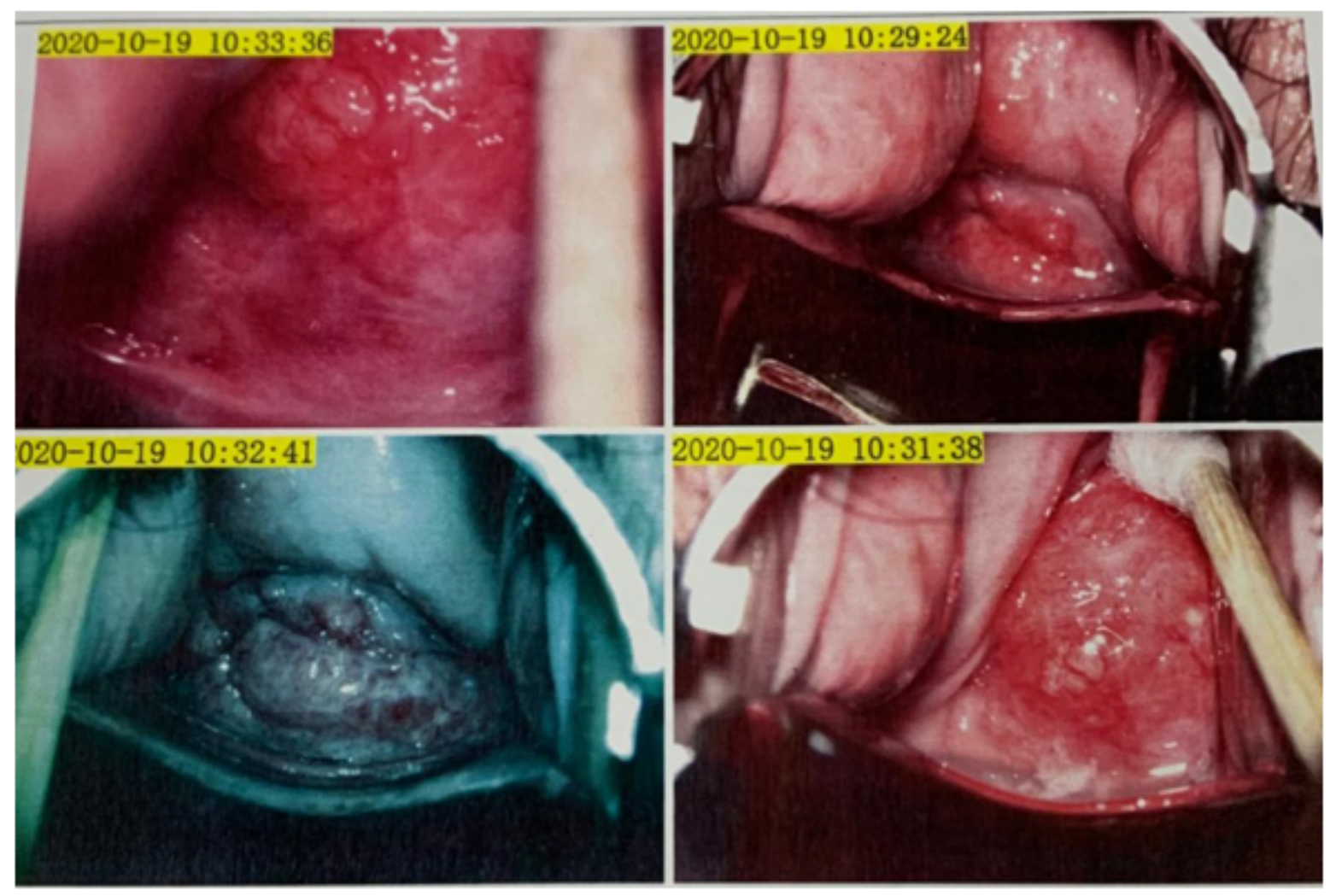

Figure 2

Cervical cancer and POP in Colposcopy. Colposcopy can be seen: full colposcopy, scale column junction is not visible, type 3 transformation area, hard texture, perineal body see old laceration, vaginal wall in the upper part of the bulge is obvious, abnormal blood vessels, thin acetic acid white epithelium, polypoid hyperplasia. Colposcopy diagnosis: cervical atypical hyperplasia. 


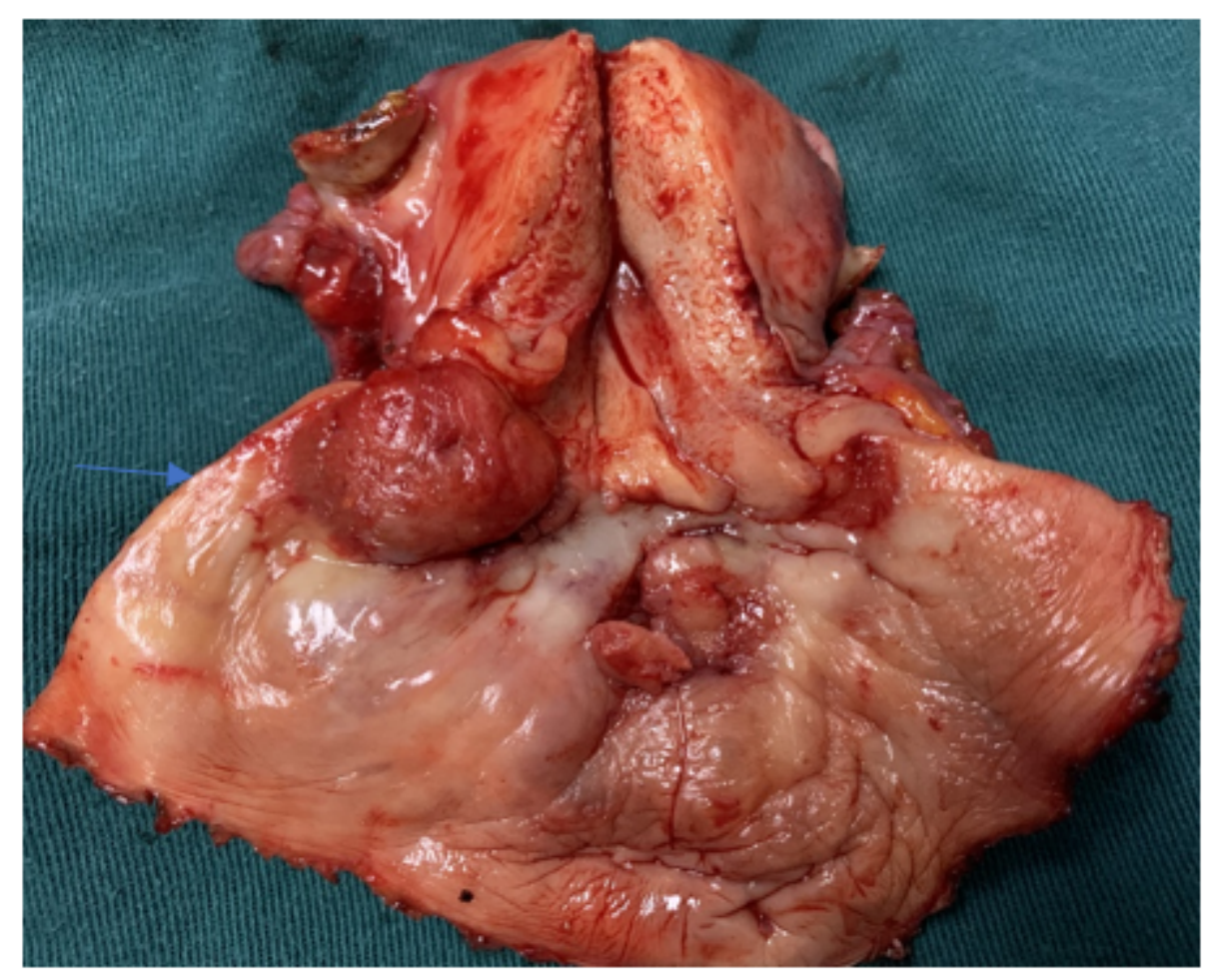

\section{Figure 3}

The specimens after radical hysterectomy of cervical cancer. The specimen was cut from the posterior wall of uterus. Biopsy showed that the anterior and posterior walls of vagina were removed $4-5 \mathrm{~cm}$. The prolapsed vagina was completely removed. The tumor (blue arrow) with a diameter of $2.5 \mathrm{~cm}$ was found in 3-4 o'clock at the outer mouth of cervix, and there was no obvious abnormal blood vessel and ulcer on the surface. 